\title{
Structurally different lysophosphatidylethanolamine species stimulate neurite outgrowth in cultured cortical neurons via distinct G-protein-coupled receptors and signaling cascades
}

\author{
Kazutoshi Hisano \\ Shinshu University \\ Shiori Kawase \\ Shinshu University \\ Tetsuhiko Mimura \\ Shinshu University \\ Hiroki Yamada \\ Shinshu University \\ Hisao Haniu \\ Shinshu University \\ Tamotsu Tsukahara \\ Nagasaki University
}

Taiga Kurihara

Nihon Parmaceutical University

Yoshikazu Matsuda

Nihon Pharmaceutical University

Naoto Saito

Shinshu University

Takeshi Uemura ( $\square$ tuemura@shinshu-u.ac.jp )

Shinshu University https://orcid.org/0000-0001-6000-4618

\section{Research Article}

Keywords: Lysophosphatidylethanolamine, Cultured cortical neuron, Neurite outgrowth, G-protein-coupled receptor, Signaling cascade

Posted Date: November 18th, 2020

DOI: https://doi.org/10.21203/rs.3.rs-74078/v2 
License: (c) (i) This work is licensed under a Creative Commons Attribution 4.0 International License. Read Full License

Version of Record: A version of this preprint was published at Biochemical and Biophysical Research Communications on January 1st, 2021. See the published version at https://doi.org/10.1016/j.bbrc.2020.11.119. 


\section{Abstract}

Neurite outgrowth is important in neuronal circuit formation and functions, and for regeneration of neuronal networks following trauma and disease in the brain. Thus, identification and characterization of the molecules that regulate neurite outgrowth are essential for understanding how brain circuits form and function and for the development of treatment of neurological disorders. In this study, we found that structurally different lysophosphatidylethanolamine (LPE) species, palmitoyl LPE (16:0 LPE) and stearoyl LPE (18:0 LPE), stimulate neurite growth in cultured cortical neurons. Interestingly, YM-254890, an inhibitor of Gq/11 protein, inhibited 16:0 LPE-stimulated neurite outgrowth but not 18:0 LPE-stimulated neurite outgrowth. In contrast, pertussis toxin, an inhibitor of Gi/Go proteins, inhibited 18:0 LPEstimulated neurite outgrowth but not 16:0 LPE-stimulated neurite outgrowth. The effects of protein kinase C inhibitors on neurite outgrowth were also different. In addition, both 16:0 LPE and 18:0 LPE activate mitogen-activated protein kinase (MAPK)/extracellular signal-regulated kinase (ERK)1/2, but the effect of the MAPK inhibitor differed between the 16:0 LPE- and 18:0 LPE-treated cultures. Collectively, the results suggest that the structurally different LPE species, 16:0 LPE and 18:0 LPE stimulate neurite outgrowth through distinct signaling cascades in cultured cortical neurons and that distinct $\mathrm{G}$ protein-coupled receptors are involved in these processes.

\section{Introduction}

Neurite outgrowth is important in neuronal wiring during development and synaptic plasticity under physiological conditions, and in the regeneration of neuronal wiring following trauma and disease in the brain $[1,2]$. Thus, identifying the molecules that regulate neurite outgrowth and elucidating their mechanism are essential for understanding of mechanisms of neuronal wiring and brain functions, and for development of therapeutics to treat trauma or neurological disorders. To date, several extracellular ligands that regulate the neurite outgrowth of central nervous system (CNS) neurons have been identified. These ligands are varied and include neurotrophins, Wnt proteins, neurotransmitters, neuropeptides [1-3].

Phospholipids are amphiphilic molecules with a hydrophilic head esterified by a hydrophobic fatty acid chain. They play crucial roles in various biological processes, ranging from structural integrity of cellular and organelle membranes, to intracellular signal transduction [4]. The brain is one of the most lipid-rich organs, and phospholipids especially play important roles not only in membrane organization but also in brain function $[5,6]$. Among the physiologically active phospholipids, the role of lysophospholipids in biological functions has attracted recent attention. Lysophospholipids consisted of a hydrophilic head group and one hydrophobic fatty acid chain, and many lysophospholipid molecular species exist. Lysophosphatidylethanolamine (LPE) is one of the lysophospholipids derived from phosphatidylethanolamine (PE) via a phospholipase A-type reaction and is known to be a minor constituent of cell membranes $[7,8]$. As for other lysophospholipids, LPE presents many structurally different species that differ in fatty acid length and degree of unsaturation [7]. Extracellularly, LPE has been detected in human serum at levels of several hundred nanograms per milliliter, but its physiological role remains largely unknown [8]. A few studies suggest the cell-type specific roles of LPE in cultures [9- 
12]. Interestingly, several studies show that cognitive impairment or traumatic brain damage in rodent model is accompanied by changes in LPE revels in the brain [13-15], suggesting the physiological or pathological significance of LPE. However, to date, the role of LPE in CNS neurons remains unclear. In this study, we demonstrate that both palmitoyl LPE (16:0 LPE) and stearoyl LPE (18:0 LPE) stimulate neurite outgrowth in the cultured cortical neurons. Moreover, using several G-proteins and enzyme inhibitors, we found that distinct G-protein-coupled receptors (GPCRs) and signaling cascades are involved in 16:0 LPE and 18:0 LPE-induced neurite outgrowth.

\section{Materials And Methods}

\subsection{Cell cultures}

Primary cortical neuron cultures were prepared from ICR mice at embryonic day 18 as described previously [16]. The cells were placed on 12-diameter coverslips coated with poly-L-lysine and laminin at a density of $1.0 \times 10^{5}$ cells / well on 24-well culture plates. For western blot analysis, primary cortical neuron cultures were plated on the 24-well dish at a density of $5.0 \times 10^{5}$ cells / well. All animal procedures were approved by the Animal Care and the Use Committee of Shinshu University (Approval No. 290072).

\subsection{Morphological analysis}

Phospholipid kit including bovine liver lysophosphatidylcholine (LPC), egg yolk LPE, soybean phosphatidylinositol (PI), bovine heart sphingomyelin (SM), porcine brain phosphatidylserine (PS), bovine liver phosphatidylcholine (PC), egg yolk phosphatidylethanolamine (PE), bovine heart cardiolipin ( $C L)$, phosphatidic acid (PA), and porcine brain cerebrosides (CB) were purchased from Olbracht Serdary Research Laboratories. At DIV3, each phospholipid was applied to the medium. The cells were fixed at DIV14 and immunostained with antibodies. For inhibitor experiments, inhibitors were applied to the culture medium at DIV0, and fixed at DIV3. Detail information is shown in Supplementary materials and methods.

\subsection{Western blot analysis}

Western blot analysis was performed using mouse anti-phospho-p44/42 mitogen-activated protein kinase (MAPK)/ERK1/2 (Thr202/Tyr204) and rabbit anti-p44/42 MAPK (ERK 1/2) antibodies (see the Supplementary materials and methods for details).

\subsection{Statistical analysis}

Results of at least 2 independent experiments were subjected to statistical analyses. Statistical significance was evaluated with two-way or one-way ANOVA followed by Tukey's, Dunnett's post hoc test, or Student's t test, or Kruskal-Wallis test followed by post-hoc Steel-Dwass or Steel's test using $\mathrm{R}$ software (R Core Team, 2017). Statistical significance was assumed when $p<0.05$. 


\section{Results}

\subsection{Egg yolk LPE increases axon and dendritic area in cultured cortical neurons}

To examine the effects of phospholipids on neuronal morphology, a commercially available phospholipid kit containing 10 different phospholipids was applied to cultured cortical neurons. In the cultured cortical neurons, microtubule-associated protein 2 (MAP2)-positive dendrites and microtubule-associated protein tau (tau)-positive axons had spread all over the dish (Fig. 1A). When the cultured cortical neurons were incubated with $1 \mu \mathrm{M}$ egg yolk LPE for 11 days, the tau and MAP2 signals area were significantly increased compared to those in the control cultures (Fig. 1A-C). These results suggest that egg yolk LPE strongly stimulates both axonal and dendritic outgrowth in cultured cortical neurons. In cultures treated with soybean PI and bovine heart PC, MAP2 and tau signals were slightly increased compared to those in the control cultures. On the other hand, there was no substantial difference in the number of neuronal nuclear antigen (NeuN)-positive neurons between the control and the cultures treated with phospholipids, except for treatment with bovine heart CL (Fig. 1A and D). In the bovine heart CL-treated cultures, the number of NeuN-positive neurons, the tau and MAP2 signal areas were significantly decreased.

\subsection{6:0 LPE and 18:0 LPE increase axon and dendritic area in cultured cortical neurons}

Among phospholipids tested, egg yolk LPE was the most effective on the morphological change. To our knowledge, no previous study examined the role of LPE in the CNS neurons. Therefore, LPE effects were examined in this study. The LPE used was derived from egg yolk and contained two structurally different LPE species, 16:0 LPE and 18:0 LPE. To examined whether 16:0 LPE or 18:0 LPE effects the neuronal morphology, cultured cortical neurons were incubation with different concentrations of egg yolk LPE, 16:0 LPE, and 18:0 LPE for 11 days. Incubation with 0.1 or $1 \mu \mathrm{M}$ 16:0 LPE significantly increased the tau and MAP2 signal areas compared to those in the control cultures (Fig. 1E-G). At a higher concentration of 10 $\mu \mathrm{M}, 16: 0$ LPE did not significantly increased these signal areas. On the other hand, egg yolk LPE and 18:0 LPE significantly increased the tau and MAP2 signal areas at all concentrations (Fig. 1E-G). There was no substantial difference in the number of NeuN-positive neurons when comparing the control and the LPEstreated cultures (Fig. 1E and H). These results suggest that the structurally distinct LPE species 16:0 LPE and 18:0 LPE both increase axonal and dendritic area in cultured cortical neurons.

\subsection{6:0 LPE and 18:0 LPE stimulate neurite outgrowth in cultured cortical neurons}

The effects of 16:0 LPE and 18:0 LPE on neurite outgrowth were examined by directly measuring the length of neurites. From DIV0 to DIV3, the lengths of the longest neurites were gradually increased in all conditions. In cultures treated with $1 \mu \mathrm{M} \mathrm{16:0} \mathrm{LPE} \mathrm{and} \mathrm{18:0} \mathrm{LPE,} \mathrm{the} \mathrm{neurites} \mathrm{were} \mathrm{significantly} \mathrm{longer}$ than those of control cultures (Fig. 2A and C). The number of neurites emerging from the soma was also increased in these cultures at DIV1 and 2 (Fig. 2A and D). On the other hand, the numbers of branches for the longest neurite in 16:0 LPE- and 18:0 LPE-treated cultures were comparable to those in the control culture (Fig. 2A and E). In terms of morphologies examined, there was no substantial difference between the 16:0 LPE- and 18:0 LPE-treated cultures. The effects of different concentrations of 16:0 LPE and 18:0 
LPE were also examined in terms of neurite outgrowth. 18:0 LPE dose-dependently increased the length of neurites as reported previously (Fig. 2B and F). At a concentration of $10 \mu \mathrm{M}$, both LPEs increased the neurite length to approximately twice as long as that in the control cultures. There was no significant difference between 16:0 LPE and 18:0 LPE in the lengths at any concentration. These results suggest that 16:0 LPE and 18:0 LPE stimulate neurite outgrowth in cultured cortical neurons and have similar effects on neuronal morphology.

\subsection{6:0 LPE and 18:0 LPE activate MAPK in cultured cortical neurons}

It has been reported that LPE activates MAPK/ERK1/2 in manner that depends on cell type $[9,11,17,18]$. However, the response of LPE in neurons remains unknown. It is known that MAPK signal cascade involved in neurite outgrowth [19]. Thus, one possibility is that 16:0 LPE and 18:0 LPE activate MAPK/ERK1/2 in the cultured cortical neurons. To examine whether this takes place, the cultured cortical neurons were incubated with 16:0 LPE or 18:0 LPE and subjected to western blot analysis. Anti-ERK1/2 antibody then detected the bands corresponding to the size of ERK $1 / 2$ in all conditions (Fig. $3 \mathrm{~A}$ ). In the absence of LPE, anti-phospho-ERK1/2 antibody detected bands corresponding to the size of phosphorylated ERK1/2, and incubation of 16:0 LPE and 18:0 LPE significantly increased those signals in a dose dependent manner (Fig. 3A and B). When the cultures were treated with MAPK inhibitor U0126 at 5 $\mu \mathrm{M}, 16: 0$ LPE- and 18:0 LPE-induced activation of MAPK/ERK1/2 was completely inhibited (Fig. 3C and D). These results suggest that both 16:0 LPE and 18:0 LPE activate MAPK/ERK1/2 in cultured cortical neurons.

\subsection{Differential effects of MAPK inhibitor on 16:0 LPE- and 18:0 LPE-stimulated neurite outgrowth}

The finding of activation of MAPK/ERK1/2 in 16:0 LPE- and 18:0 LPE-treated cortical cultures rises the possibility that these LPEs stimulate neurite outgrowth through the activation of MAPK/ERK $1 / 2$. To examine this, the cultured cortical neurons were treated with MAPK inhibitor U0126 at $5 \mu \mathrm{M}$ in the presence or absence of 16:0 LPE or 18:0 LPE for 3 days. In the control cultures at DIV3, application of 5 $\mu \mathrm{M}$ U0126 significantly decreased the length of neurites. In cultures treated with 18:0 LPE, U0126 significantly decreased the length of neurite to a degree comparable to that in the U0126-treated control cultures (Fig. 3E and F). In contrast, U0126 decreased 16:0 LPE-stimulated neurite length, although these were longer than in the control U0126-treated, and 18:0 LPE and U0126-treated cultures. These results suggest that 16:0 LPE and 18:0 LPE stimulate neurite outgrowth through the activation of MAPK. In addition, the difference effect of MAPK inhibitor on 16:0 LPE- and 18:0 LPE-stimulated neurite outgrowth raises the possibility that these LPEs activate distinct membrane receptors and signaling cascades.

\subsection{Differential effects of G-protein inhibitors on 16:0 LPE-and 18:0 LPE-stimulated neurite outgrowth}

Several studies suggest that the one of actions of LPE is mediated by GPCR-activated G protein-coupled PLC $[10,11,20-22]$. To examined whether the GPCR-PLC cascade mediates the actions of 16:0 LPE and 18:0 LPE, cultured cortical neurons were incubated with Gq/11 inhibitor YM-254890 or Gi/Go inhibitor pertussis toxin (PTX). Application of YM-254890 decreased the length of 16:0 LPE-stimulated neurite, but 
not that of 18:0 LPE-stimulated neurite (Fig. 4A an F). In contrast, PTX decreased the length of 18:0 LPEstimulated neurite, but not that of 16:0 LPE-stimulated neurite (Fig. 4B an G). PLC inhibitor U73122 decreased both the length of 16:0 LPE- and 18:0 LPE-stimulated neurite (Fig. $4 \mathrm{C}$ and $\mathrm{H}$ ). It is known that activation of PLC leads to production of inositol 1,4,5-trisphosphate and diacylglycerol, the activator for PKC. To examine whether PKC is involved in LPEs-stimulated neurite outgrowth, the PKC inhibitor Go6983, which inhibits PKCa, $\beta, y, \delta$, and $\zeta$ [23], was applied to the cultures. Application of $1 \mu \mathrm{M}$ Go6983 inhibited 18:0 LPE-stimulated neurite outgrowth. In contrast, Go6983 had little effect on 16:0 LPE-treated cultures (Fig. 4D and I). It is known that PKC family proteins consist of PKCa, $\beta, \gamma, \delta, \varepsilon, \eta, \theta, \zeta, M \zeta$, and $\mathrm{t} / \lambda$ [24]. Therefore, a different PKC inhibitor, Sotrastaurin, which inhibits PKCa, $\beta, \delta, \varepsilon, \eta$, and $\theta$ [25], was selected. When Sotrastaurin was applied to the cultures, both 16:0 LPE- and 18:0 LPE-stimulated neurite length changes were completely inhibited (Fig. $4 \mathrm{E}$ and J). These results suggest that 16:0 LPE and 18:0 LPE activate distinct GPCRs and signaling cascades involved in the neurite outgrowth.

\section{Discussion}

Recently, the role of lysophospholipids in biological functions has attracted attention. However, in the CNS neurons, the cellular response to extracellular LPE remains unknown. In this study, we found that extracellular application of 16:0 LPE and 18:0 LPE stimulates neurite outgrowth in cultured cortical neurons and that distinct GPCRs and signaling cascades are involved in these processes.

The effects of 16:0 LPE and 18:0 LPE in stimulating neurite outgrowth in cultured cortical neurons are clearly shown (Fig. 2). To date, a few studies have indicated the cell-type specific roles of LPE in culture cells, but physiological role remains largely unknown. The LPE extracted from Grifola frondosa, a kind of mushroom, stimulates neurite-like outgrowth of rat pheochromocytoma PC12 cells [9]. The 18:1 LPE stimulates chemotactic migration and cellular invasion in SK-OV3 human ovarian cancer cells [10] and cell proliferation of human MDA-MB-231 breast cancer cells [11]. Egg yolk LPE stimulates differentiation and maturation of mouse astrocyte in culture [12]. Thus, LPE evokes various response, depending on the cell type. At this time, whether LPE promotes neurite outgrowth in other types of CNS neurons remains unclear.

In cultured cortical neurons, 16:0 LPE-stimulated neurite outgrowth was inhibited by Gq/11 inhibitor YM254890 and PLC inhibitor U73122 (Fig. 4), suggesting that the action of 16:0 LPE is mediated by Gq/11coupled GPCR activated PLC. On the other hand, 18:0 LPE-stimulated neurite outgrowth was inhibited by Gi/Go inhibitor PTX and U73122(Fig. 4), suggesting that the action of 18:0 LPE is mediated Gi/Gocoupled GPCR activated PLC [26]. Thus, different types of GPCRs are likely to exist for 16:0 LPE and 18:0 LPE. To date, receptors for LPE remains unidentified, but several studies have suggested the existence of cell type-specific GPCRs for different LPE species [10,11,20-22]. For example, in MDA-MB-231cells, 18:1 LPE stimulates intracellular $\mathrm{Ca}^{2+}$ concentration, and this effect is inhibited by PTX or LPA receptor LPA antagonist AM095 [11,20]. On the other hand, in SK-OV3 cells, the effect of 18:1 LPE-stimulated intracellular $\mathrm{Ca}^{2+}$ concentration is inhibited by PTX, but not by AM095 [10, 11]. In addition, in some cells, LPE stimulates intracellular $\mathrm{Ca}^{2+}$ concentration, but their effect is completely dependent on the fatty acid 
lengths of the LPE $[10,11,20-22]$. For example, in PC12 cells, intracellular $\mathrm{Ca}^{2+}$ concentration is stimulated by 18:0 LPE and 14:0 LPE, but not 16:0 LPE [21]. Identification of receptors for 16:0 LPE and 18:0 LPE will be needed to completely elucidate how LPE induces a signal cascade in the cortical neuron.

Many ligands including neurotrophins, Wnt proteins, neurotransmitters, and neuropeptides have been identified as stimulating neurite outgrowth of neurons via binding to their receptors [1-3], and diverse protein kinase signal cascades including MAPK and PKC are involved in this process [19]. In the cultured cortical neurons, extracellular application of both 16:0 LPE and 18:0 LPE activated MAPK/ERK1/2 (Fig. 3). It has reported that 14:0 LPE, 16:0 LPE, and 18:1 LPE activate MAPK/ERK1/2 in PC12 cells [9] and 18:1 LPE activates ERK1/2 in human MDA-MB-231 breast cancer cell line [11]. LPE, although not reported in terms of specific type, did not activate MAPK in murine mesangial cells [18]. However, for PC12 cells, contrary to the observations noted by Nishina et al [9], there is a report that 16:0 LPE does not activate MAPK/ERK1/2 in these cells [17]. These previous studies suggest the existence of a cell-type dependent MAPK activation mechanism for LPEs. Interestingly, in cultured cortical neurons, inhibition of MAPK inhibited 18:0 LPE-stimulated neurite outgrowth and partially inhibited 16:0 LPE-stimulated neurite outgrowth (Fig.3). In addition, Sotrastaurin, which inhibits PKCa, $\beta, \delta, \varepsilon, \eta$, and $\theta$ [25], inhibited both 16:0 LPE- and 18:0 LPE-induced neurite outgrowth. In contrast, Go6983, which inhibits PKCa, $\beta, \gamma, \delta$, and $\zeta$ [23], inhibited the effect of 18:0 LPE but not the effect of 16:0 LPE (Fig. 4D, E, I and J). Combining these results with the G-proteins inhibitor results (Fig. 4A, B, F, and G) suggest the involvement of distinct GPCRs and signaling cascades in 16:0 LPE and 18:0 LPE-induced neurite outgrowth.

Thus far, the roles of LPE in the brain has not been fully elucidated, but several reports suggest the pathophysiological significance of LPE. Previous reports show that cognitive impairment or traumatic brain damage is associated with an imbalance in the composition of phospholipids in the brain, such that some studies have reported changes of LPE level in the brain. Aged triple transgenic Alzheimer's model mice harboring PS1M146V, APPswe, and tau P301L transgenes with cognitive impairment show increasement of LPE in the hippocampus [13]. Postischemic cognitive impairment rat model show increasement of LPE species, 18:1, 20:3, and 22:6 LPE, in the hippocampus [15]. Traumatic brain injury in rat transiently increases 22:6 LPE in the injury area [14]. A change in LPE was reported in the plasma of a major depressive disorder patient [27]. These observations imply the importance of LPE in the brain in normal development, as well as in pathological setting. Comprehensive analysis of LPE species in the brain and investigation of their functions are necessary to understand their roles in pathophysiological conditions.

\section{Declarations}

\section{Competing interest}

The authors declare that they have no conflict of interest.

\section{Acknowledgements}


This work was supported by JSPS KAKENHI Grant Number JP19H03334 to T.U. and JP20K09476 to H.H.

\section{References}

[1] P.J. Horner, F.H. Gage, Regenerating the damaged central nervous system, Nature 407 (2000) 407:963970.

[2] E,T. Stoeckli, Understanding axon guidance: are we nearly there yet?, Development 145 (2018) $\operatorname{dev} 151415$.

[3] A.B. Huber, A.L. Kolodkin, D.D. Ginty, et al., Signaling at the growth cone: ligand-receptor complexes and the control of axon growth and guidance. Annu. Rev. Neurosci. 26 (2003) 509-563.

[4] G. Van Meer, D.R. Voelker, G.W. Feigenson, Membrane lipids: where they are and how they behave, Nat. Rev. Mol. Cell Biol. 9 (2008) 112-124.

[5] T.J. Tracey, F.J. Steyn, E.J. Wolvetang, et al., Neuronal lipid metabolism: multiple pathways driving functional outcomes in health and disease, Front. Mol. Neurosci.11 (2018) 10.

[6] J.W. Choi, J. Chun, Lysophospholipids and their receptors in the central nervous system, Biochim. Biophys. Acta. 1813 (2013) 20-32.

[7] W.J. Van Blitterswijk, G. De Veer, J.H. Krol, et al., Comparative lipid analysis of purified plasma membranes and shed extracellular membrane vesicles from normal murine thymocytes and leukemic GRSL cells, Biochim. Biophys. Acta. 688 (1982) 495-504.

[8] K. Makide, H. Kitamura, Y. Sato, et al., Emerging lysophospholipid mediators, lysophosphatidylserine, lysophosphatidylthreonine, lysophosphatidylethanolamine and lysophosphatidylglycerol, Prostaglandins. Other Lipid Mediat. 89 (2009) 135-139.

[9] A. Nishina, H. Kimura, A. Sekiguchi, et al., Lysophosphatidylethanolamine in Grifola frondosa as a neurotrophic activator via activation of MAPK, J. Lipid Res. 47 (2006) 1434-1443.

[10] K.S. Park, H.Y. Lee, S.Y. Lee, et al., Lysophosphatidylethanolamine stimulates chemotactic migration and cellular invasion in SK-OV3 human ovarian cancer cells: involvement of pertussis toxin-sensitive Gprotein coupled receptor, FEBS Lett. 581 (2007) 4411-4416.

[11] S.J, Park, K.P. Lee, D.S. Im, Action and signaling of lysophosphatidylethanolamine in MDA-MB-231 breast cancer cells, Biomol. Ther. (Seoul) 22 (2014) 129-135.

[12] A. Montaner, T.T. da Silva Santana, T. Schroeder, et al., Specific phospholipids regulate the acquisition of neuronal and astroglial identities in post-mitotic cells, Sci Rep. 8 (2018) 460. 
[13] J.G. Villamil-Ortiz, A. Barrera-Ocampo, D. Piedrahita, et al., BACE1 RNAi restores the composition of phosphatidylethanolamine-derivates related to memory improvement in Aged 3xTg-AD mice, Front Cell. Neurosci. 10 (2016) 260.

[14] S. Guo, D. Zhou, M. Zhang, et al., Monitoring changes of docosahexaenoic acid-containing lipids during the recovery process of traumatic brain injury in rat using mass spectrometry imaging, Sci. Rep. 7 (2017) 5054.

[15] A.M. Sabogal-Guáqueta, J.G. Villamil-Ortiz, J.D. Arias-Londoño, et al., Inverse phosphatidylcholine/phosphatidylinositol levels as peripheral biomarkers and phosphatidylcholine/lysophosphatidylethanolamine-phosphatidylserine as hippocampal indicator of postischemic cognitive impairment in rats, Front. Neurosci. 12 (2018) 989.

[16] T. Uemura, T. Shiroshima, A. Maeda, et al, In situ screening for postsynaptic cell adhesion molecules during synapse formation, J Biochem. 162 (2017) 295-302.

[17] Wuhanqimuge, A. Itakura, Y. Matsuki, M. et al., Lysophosphatidylcholine enhances NGF-induced MAPK and Akt signals through the extracellular domain of TrkA in PC12 cells, FEBS Open Bio. 3 (2013) 243-251.

[18] B.V. Bassa, D.D. Roh, N.D. Vaziri, et al., Lysophosphatidylcholine activates mesangial cell PKC and MAP kinase by PLCgamma-1 and tyrosine kinase-Ras pathways, Am. J. Physiol. 277 (1999) F328-337.

[19] S.A. Bennison, S.M. Blazejewski, T.H. Smith, et al., Protein kinases: master regulators of neuritogenesis and therapeutic targets for axon regeneration, Cell. Mol. Life Sci. 77 (2020) 1511-1530.

[20] S.J. Park, K.P. Lee, S. Kang, et al., Lysophosphatidylethanolamine utilizes LPA $_{1}$ and CD97 in MDA-MB231 breast cancer cells, Cell Signal. 25 (2013) 2147-2154.

[21] J.M. Lee, S.J Park, D.S. Im, Lysophosphatidylethanolamine increases intracellular $\mathrm{Ca}^{2+}$ through $\mathrm{LPA}_{1}$ in PC-12 neuronal cell, Biochem. Biophys. Res. Commun. 461 (2015) 378-382.

[22] J.M. Lee, S.J. Park, D.S. Im, Calcium signaling of lysophosphatidylethanolamine through $\mathrm{LPA}_{1}$ in human SH-SY5Y neuroblastoma cells, Biomol Ther (Seoul). 25 (2017) 194-201.

[23] M. Gschwendt, S. Dieterich, J. Rennecke, et al., Inhibition of protein kinase $\mathrm{C} \mu$ by various inhibitors. Differentiation from protein kinase c isoenzymes, FEBS Lett. 392 (1996) 77-80.

[24] S.F. Steinberg, Structural basis of protein kinase C isoform function, Physiol. Rev. 88 (2008) 13411378.

[25] J.P. Evenou, J. Wagner, G. Zenke, et al., The potent protein kinase C-selective inhibitor AEB071 (sotrastaurin) represents a new class of immunosuppressive agents affecting early T-cell activation, $\mathrm{J}$. Pharmacol. Exp. Ther. 330 (2009) 792-801. 
[26] B. Hille, G protein-coupled mechanisms and nervous signaling, Neuron. 9 (1992) 187-195.

[27] X. Liu, J. Li, P. Zheng, et al., Plasma lipidomics reveals potential lipid markers of major depressive disorder, Anal. Bioanal. Chem. 408 (2016) 6497-6507.

\section{Figures}
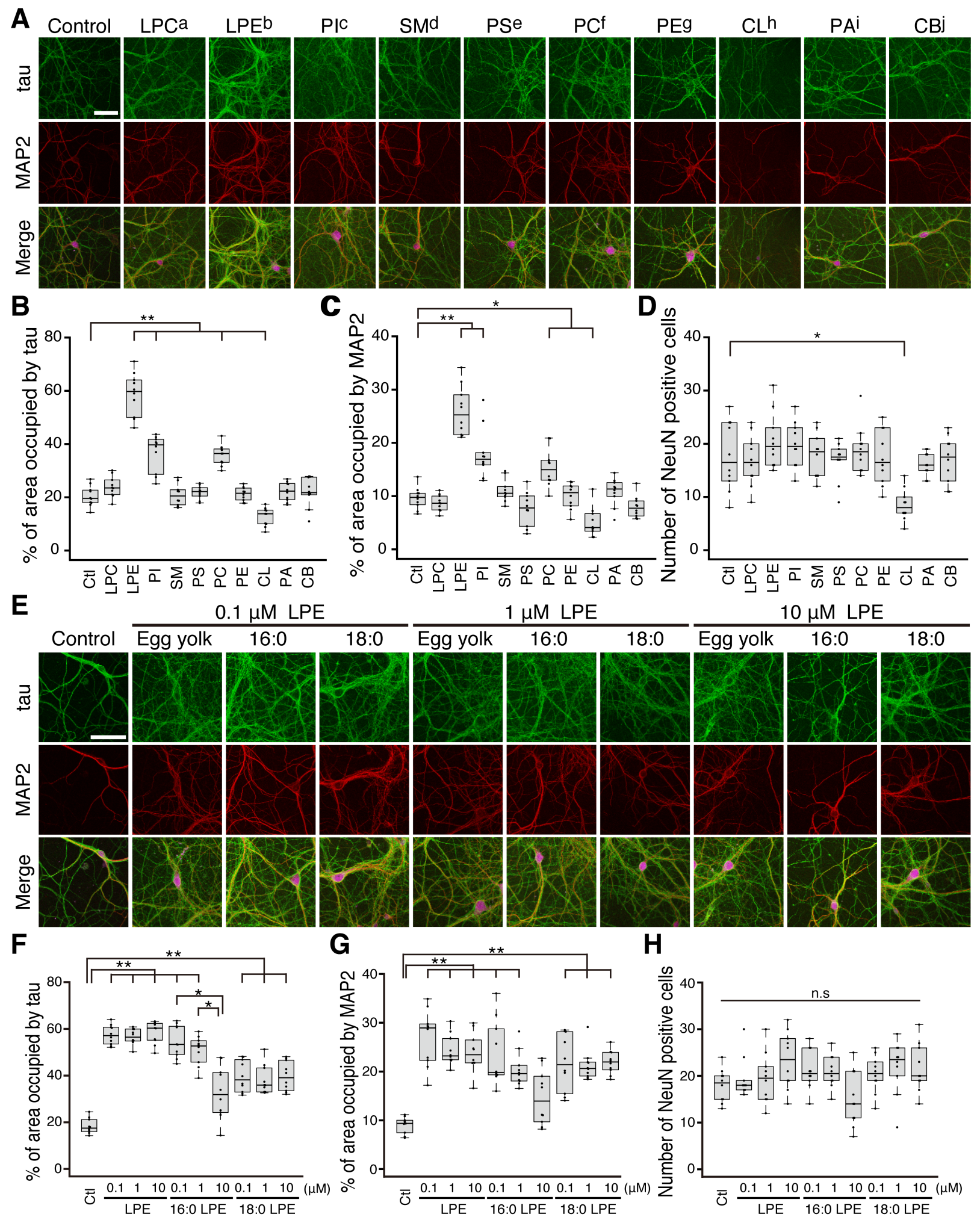
Effects of phospholipids on neuronal morphology. (A) Extracellular application of egg yolk LPE, soybean $\mathrm{Pl}$, or bovine liver PC increased the MAP2 and tau staining signals in the cultured cortical neurons. tau (green), MAP2 (red), and NeuN (magenta) signals are merged on the bottom. abovine liver LPC; begg yolk LPE; csoybean Pl; dbovine heart SM; eporcine brain PS; fbovine liver PC; gegg yolk PE; hbovine heart CL; iPA form egg yolk PC; jporcine brain CB. (B, C) Quantification of tau (B) and MAP2 (C) signals area in (A). The percentage of tau and MAP2 signals occupied in in the observation field were measured. (D) Quantification of number of NeuN positive neurons in (A). (E) Extracellular application of 16:0 LPE and 18:0 LPE increased the MAP2 and tau staining signals. tau (green), MAP2 (red), and NeuN (magenta) signals are merged on the bottom. $(F, G)$ Quantification of tau $(F)$ and MAP2 $(G)$ signals area in $(E)$. The percentage of tau and MAP2 signals occupied in in the observation field were measured. $(H)$ Quantification of number of NeuN positive neurons in (E). Scale bars represent $50 \mu \mathrm{m}$. The horizontal line in each box indicates the median, the box shows the interquartile range (IQR), and the whiskers are 1.5x IQR. Statistical significance was evaluated with the Kruskal-Wallis test followed by post hoc Steel test in $(B-D)$ or Steel-Dwass test in $(F-H)$ : ** $p<0.01$ and * $p<0.05, n=10$ areas.

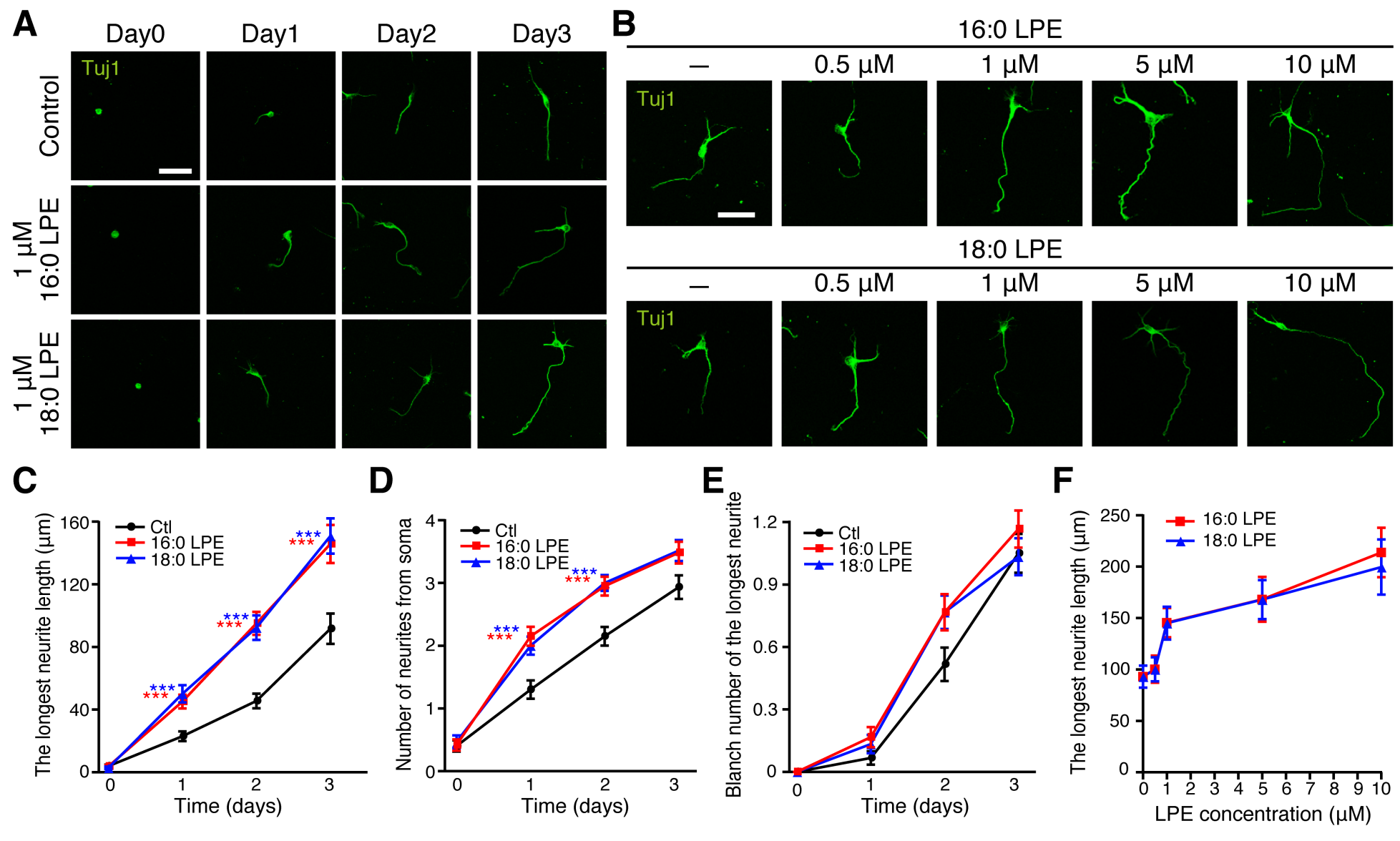

Figure 2

Effects of 16:0 LPE and 18:0 LPE on neurite outgrowth. (A) Extracellular application of 16:0 LPE and 18:0 LPE stimulates neurite outgrowth of the cultured cortical neurons. (B) Dose-dependent stimulation of neurite outgrowth by application of 16:0 LPE and 18:0 LPE. (C) Quantification of the length of the longest 
neurite emerging from the soma in (A). (D) Quantification of the number of neurites emerging from the soma in (A). (E) Quantification of the numbers of branches per longest neurite in (A). (F) Quantification of the length of the longest neurite emerging from the soma in (B). Scale bars represent $50 \mu \mathrm{m}$. All values represent mean \pm s.e.m. Statistical significance was evaluated with Kruskal-Wallis test followed by post hoc Steel-Dwass test in (C-E) or evaluated with two-way ANOVA (LPE species $\times$ LPE concentration interaction, $F(1,5)=755.0251, P=2.2 \times 10-16)$ followed by post hoc Student's $t$ test in $(F)$ : $* \star \star ~ p<0.001$, $n=60$ cells in $(C-E), n=20$ cells in $(F)$.
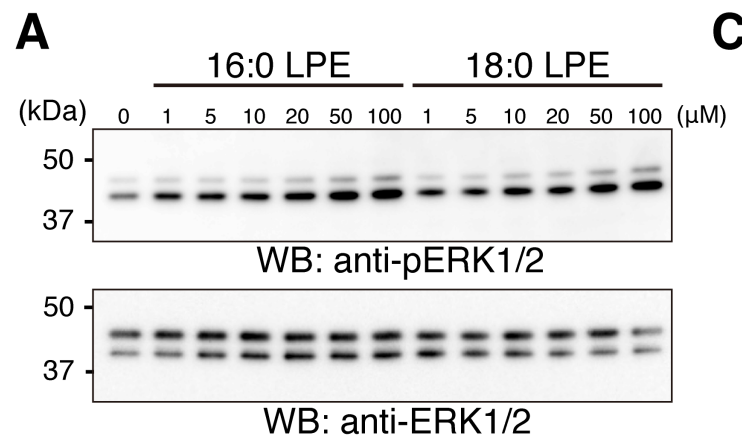

B
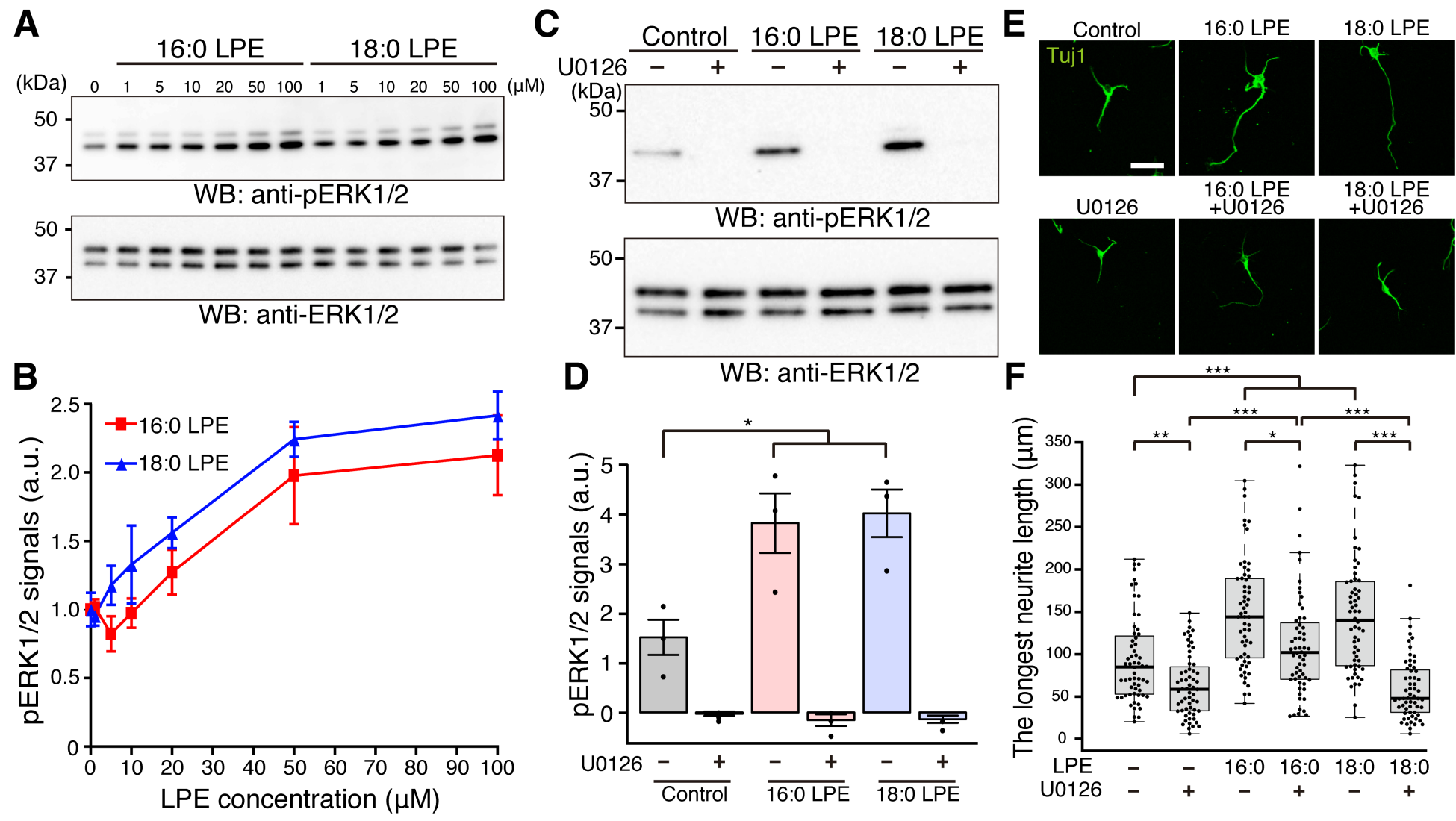

Figure 3

Activation of MAPK in 16:0 LPE- and 18:0 LPE-treated cortical cultures. (A) Activation of MAPK/ERK1/2 by 16:0 LPE and 18:0 LPE in cultured cortical neurons. (B) Quantification of phosphorylated ERK1/2 signals in (A). (C) U0126 inhibits 16:0 LPE- or 18:0 LPE-induced phosphorylation of ERK1/2. The cultures were incubated with $100 \mu \mathrm{M}$ 16:0 LPE or 18:0 LPE, with or without $5 \mu \mathrm{M}$ U0126. (D) Quantification of phosphorylated ERK1/2 signals in (C). (E) Effects of MAPK inhibitor U0126 on 16:0 LPEand 18:0 LPE-stimulated neurite outgrowth. LPEs at $1 \mu \mathrm{M}$ were applied to the cultures with or without 5 $\mu \mathrm{M}$ U0126. Scale bar represents $50 \mu \mathrm{m}$. (F) Quantification of the length of the longest neurite in (E). All values represent mean \pm s.e.m in $(B, D)$. The horizontal line in each box indicates the median, the box shows the IQR, and the whiskers are 1.5 $\times$ IQR in $(F)$. Statistical significance was evaluated using two-way ANOVA (LPE species $\times$ LPE concentration, $F(1,6)=850.7656, P=2.2 \times 10-16)$ followed by post hoc Student's $t$ test in (B), one-way ANOVA followed by post hoc Tukey's test in (D), or the Kruskal-Wallis test followed by post hoc Steel-Dwass test in $(F)$. ${ }^{* *} p<0.001$, ${ }^{* *} p<0.01$, and $* p<0.05, n=3$ cultures in (B, D) and $n=60$ cells in $(F)$, respectively. 


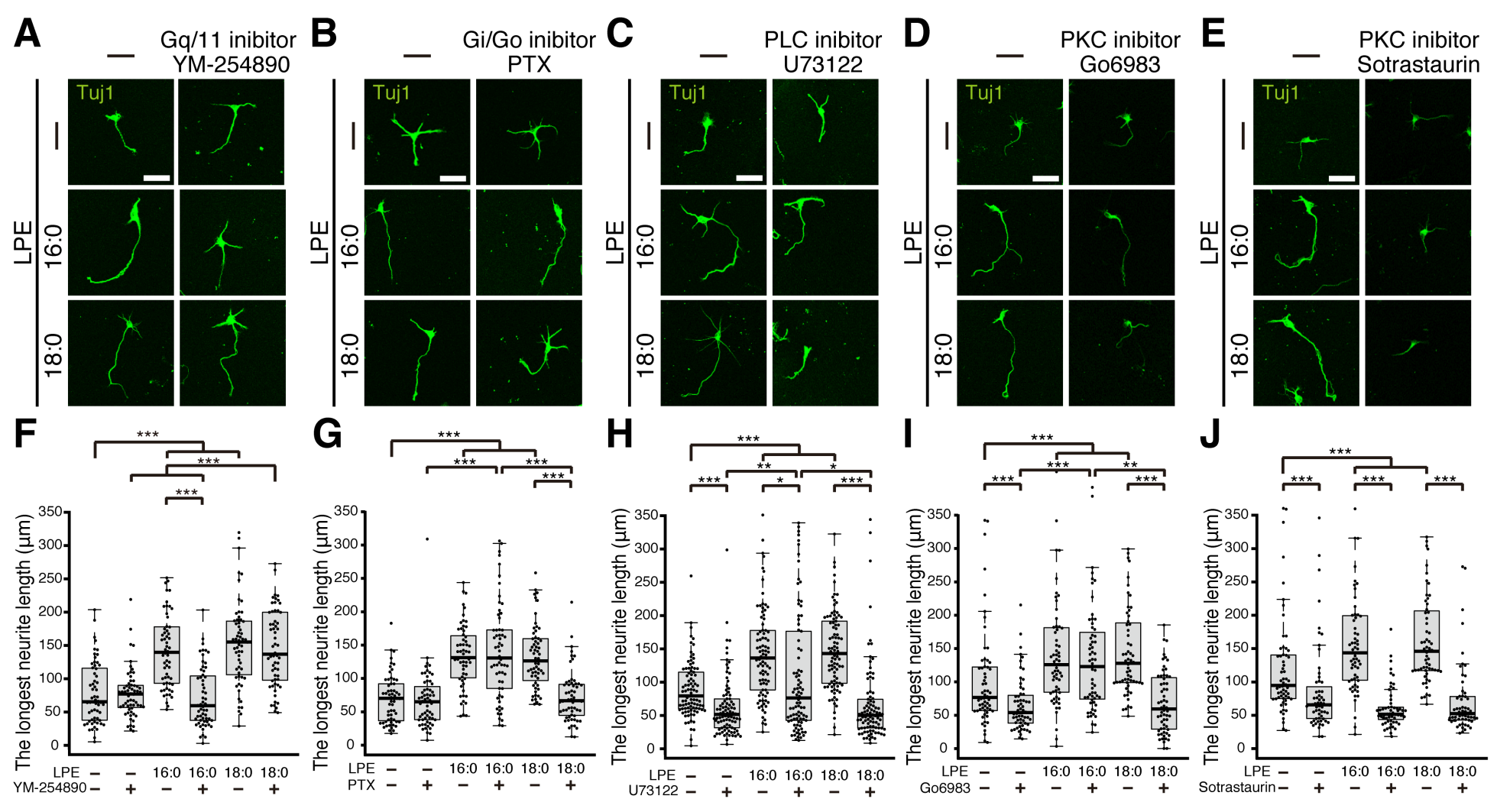

\section{Figure 4}

Effects of inhibitors for G-proteins and their downstream signal cascades. (A-E) Effects of Gq/11 inhibitor YM-254890 (A), Gi/Go inhibitor PTX (B), PLC inhibitor U73122 (C), PKC inhibitor Go6983 (D), and PKC inhibitor Sotrastaurin (E) on 16:0 LPE- and 18:0 LPE-stimulated neurite outgrowth. (F-J) Quantification of the length of the longest neurite in $(A-E)$. The horizontal line in each box indicates the median, the box shows the IQR, and the whiskers are 1.5× IQR. Statistical significance was evaluated with the Kruskal-Wallis test followed by post hoc Steel-Dwass test: ${ }^{\star \star \star} p<0.001,{ }^{\star \star} p<0.01,{ }^{\star} p<0.05, n=$ 60 cells in (F, G, I, and J), $n=90$ cells in $(H)$.

\section{Supplementary Files}

This is a list of supplementary files associated with this preprint. Click to download.

- SupplementaryinformationHisanoetal2020.10.20.docx 